\title{
On the role of coupling in mode selective excitation using ultrafast pulse shaping in stimulated Raman spectroscopy
}

\author{
S. A. Malinovskaya, P. H. Bucksbaum, and P. R. Berman \\ Michigan Center for Theoretical Physics, FOCUS Center, and Department of Physics, \\ University of Michigan, Ann Arbor, Michigan 48109
}

(Received 3 May 2004; accepted 1 June 2004)

\begin{abstract}
The coherence of two coupled two-level systems, representing vibrational modes in a semiclassical model, is calculated in weak and strong fields for various coupling schemes and for different relative phases between initial state amplitudes. A relative phase equal to $\pi$ projects the system into a dark state. The selective excitation of one of the two, two-level systems is studied as a function of coupling strength and initial phases. (C) 2004 American Institute of Physics.
\end{abstract}

[DOI: $10.1063 / 1.1774985$ ]

\section{INTRODUCTION}

Investigations of coherent dynamics in multidimensional systems induced by ultrafast shaped pulses are being carried out by a number of research groups. ${ }^{1-9}$ Special attention is paid to the development of femtosecond laser techniques for control over molecular motion in stimulated Raman spectroscopy. ${ }^{3,5,6,10,11}$ In these works the selective Raman excitations are achieved with femtosecond laser pulses with spectral or phase modulation. The goal is to prepare a specific quantum state to facilitate "unusual" structural changes or chemical reactions. ${ }^{8,9,12,13}$ Another fundamental application is related to the development of quantum memory for information storage. ${ }^{14} \mathrm{~A}$ composition of two-level systems, e.g., vibrational normal modes within an ensemble of molecules, driven by an optical field, may serve as a storage device of quantum information.

One of the important steps needed for efficient control of molecular motion is an understanding of the factors that govern the system's time evolution. In this paper femtosecond pulse shaping is discussed that allows for the selective excitation of unresolved, coupled Raman transitions.

In Ref. 5, a comparative analysis of Raman spectra of liquid methanol and a mixture of benzene and deuterated benzene showed experimental evidence for the dependence of the selective excitation on intramolecular coupling of normal vibrational modes in a molecule. In this paper a semiclassical model is developed for the interaction of a shaped ultrafast laser pulse with two coupled two-level systems, representing coupled vibrational modes in a single molecule. Specific questions about the mechanisms of interaction of an external field with molecular media are addressed such as how the coupling via an external field influences the controllability of selective excitation and how the result depends on the coupling strength. We also investigate the ways of implementing a coupling mechanism for coherent control. Within our model selective, high-level coherence can be achieved in two coupled two-level systems by a choice of the relative phase of the initially populated states. This phase influences the time evolution of complex probability amplitudes of a coherent superposition of states. The resultant state coher- ence, built by intensive pump pulse, determines the induced polarization in a molecular medium and the amplitudes of Raman fields generated from this coherence using a probe field. For a particular relative phase of the initially populated states, a dark state is formed with zero eigenvalue of the interaction Hamiltonian. It is known that for a quantum system in a dark state the prevention of decoherence may be achieved, e.g., Ref. 15. Molecules prepared in such a state may be useful for quantum computation and communication.

\section{BASIC FORMALISM}

A semiclassical model is used to describe the interaction of an ultrafast laser pulse with a molecular medium using stimulated Raman scattering. The model is represented schematically in Fig. 1, where two, two-level systems describe two Raman active modes in a molecule. Levels $|1\rangle$ and $|3\rangle$ are at zero relative energy, while levels $|2\rangle$ and $|4\rangle$ have energies $\hbar \omega_{2}$ and $\hbar \omega_{4}$, respectively. Transition dipole moment matrix elements of the levels with a virtual intermediate state $|b\rangle$ are equal to $\mu_{i b}$. Generally they may be different. We investigate the effects in weak and strong fields caused by coupling between normal vibrational modes in a molecule and by the relative phase of the amplitudes of the initially populated states. Transition matrix elements of the 3-4 twolevel system are assumed to be equal, $\mu_{3 b}=\mu_{4 b}$, and transition matrix elements of the 1-2 two-level system satisfy the conditions $\mu_{1 b} / \mu_{3 b}=\mu_{2 b} / \mu_{3 b}=r$. The equations of motion for the probability amplitudes of two coupled two-level systems are

$$
i \frac{d}{d t}\left(\begin{array}{c}
a_{1} \\
a_{2} \\
a_{3} \\
a_{4}
\end{array}\right)=-\chi\left(\begin{array}{cccc}
r^{2} & r^{2} & r & r \\
r^{2} & r^{2}-\frac{\omega_{2}}{\chi} & r & r \\
r & r & 1 & 1 \\
r & r & 1 & 1-\frac{\omega_{4}}{\chi}
\end{array}\right)\left(\begin{array}{c}
a_{1} \\
a_{2} \\
a_{3} \\
a_{4}
\end{array}\right),
$$

where $\chi$ is a time-dependent variable equal to $\left(\left|\mu_{3 b}\right|^{2} / 4 \hbar^{2} \Delta\right) I(t), I(t)$ is the pulse intensity envelope, and 


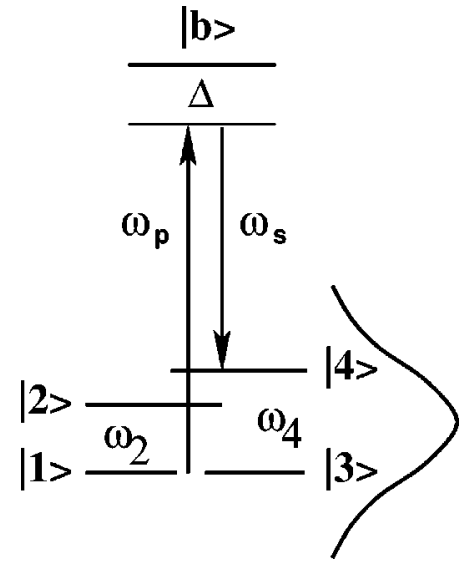

FIG. 1. Schematic picture of a model system consisting of two, two-level systems having frequencies $\omega_{2}$ and $\omega_{4}$. Initially, the lower levels are populated evenly. The transitions between four levels are driven by an offresonant femtosecond pulse.

$\Delta$ is the detuning of the frequency of the pulse from the frequency of the virtual state $|b\rangle$. Equation (1) has been obtained by adiabatic eliminating state $|b\rangle$ within the rotating wave approximation. The chosen form of the Hamiltonian allows for an adequate description of various coupling schemes. Note that in our model the pulse intensity envelope $I(t)$ is the same for all transitions. The specific form for the pulse shape is taken such that in the weak field regime the pulse selectively excites transitions of predetermined frequencies, while in strong fields the result depends on the intensity of the field $I_{0}$. The intensity envelope $I(t)$ is defined as a real part of the Fourier transform of a function $f(\omega)$ specified as

$$
f(\omega)=I_{0}\left[e^{-\alpha \omega^{2}}-e^{-\left(\omega-\omega_{4}\right)^{2} T^{2}}\left(1-e^{-\left(\omega-\omega_{2}\right)^{2} T_{1}^{2}}\right)\right],
$$

where $\alpha, T$, and $T_{1}$ are free parameters.

The real part of the Fourier transform of the spectral density (2) gives a temporal pulse function

$$
\begin{aligned}
& I(t)=I_{0} C\left\{1-A / C \cos \left(\omega_{4} t\right)\right. \\
& \left.+B / C \cos \left[\left(\omega_{2}-\Delta \omega \frac{T^{2}}{\tau^{2}}\right) t\right]\right\}, \\
& A=(\sqrt{2} T)^{-1} e^{-t^{2} / 4 T^{2}}, \\
& B=(\sqrt{2} \tau)^{-1} e^{-\Delta \omega^{2} T^{2}\left(1-T^{2} / \tau^{2}\right)-t^{2} / 4 \tau^{2},} \\
& C=(\sqrt{2} \tau)^{-1} e^{-t^{2} / 4 \tau^{2}}, \\
& \tau^{2}=T^{2}+T_{1}^{2}, \quad \Delta \omega=\omega_{4}-\omega_{2} .
\end{aligned}
$$

The temporal profile of the pulse function is shown in Fig. 2(a) for parameters corresponding to experimental data on the molecular gas $\mathrm{CO}_{2},{ }^{5} T=3, T_{1}=3$, and $\omega_{2}=1, \omega_{4}=1.1$, where $\omega_{i}$ are in frequency units, and $T, T_{1}$ are in inverse frequency units. The chosen parameters $T, T_{1}$ give a pulse duration corresponding to an impulsive regime of interaction. ${ }^{6}$ In Fig. 2(b) the Fourier transform $\widetilde{I}(\omega)$ of Eq.

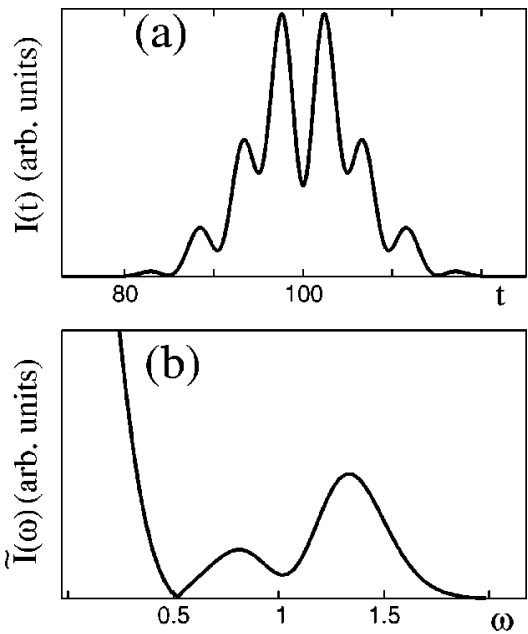

FIG. 2. (a) Intensity temporal profile $I(t)$ for $T=3, T_{1}=3\left[\omega_{2}^{-1}\right]$. (b) Fourier transform of $I(t)$.

(3) is presented showing significantly larger intensity at frequency $\omega_{4}$ than at $\omega_{2}$. In weak fields the solution of Eq. (1) is

$$
\begin{aligned}
& a_{4}=i \frac{\mu_{4 b} \mu_{3 b}^{*}}{4 \Delta \hbar^{2}} \int_{-\infty}^{\infty} I(t) e^{i \omega_{4} t} d t=i \frac{\mu_{4 b} \mu_{3 b}^{*}}{4 \Delta \hbar^{2}} \widetilde{I}\left(\omega_{4}\right), \\
& a_{2}=i \frac{\mu_{2 b} \mu_{1 b}^{*}}{4 \Delta \hbar^{2}} \int_{-\infty}^{\infty} I(t) e^{i \omega_{2} t} d t=i \frac{\mu_{2 b} \mu_{1 b}^{*}}{4 \Delta \hbar^{2}} \widetilde{I}\left(\omega_{2}\right) .
\end{aligned}
$$

As a consequence, when $I(t)$ is used in Eq. (1), the solution in a perturbative limit leads to a weak excitation of coherence $\left|\rho_{12}\right|$, and an efficient excitation of coherence $\left|\rho_{34}\right|$ with the magnitude proportional to $\widetilde{I}\left(\omega_{4}\right)$.

In strong fields the exact numerical solution of Eq. (1) shows that a choice of the field strength parameter $I_{0}$ provides control over excitations, resulting in maximum coherence for either $\left|\rho_{34}\right|$ or $\left|\rho_{12}\right|$. These results are in agreement with those published in Ref. 16 for two uncoupled two-level systems.

\section{MODE COUPLING}

A numerical solution of the time-dependent Schrödinger equation (1) describing two coupled two-level systems was obtained with $I(t)$ given by Eq. (3) and two values of $r$, equal to $\frac{1}{2}$ and 1 . The results reveal the importance of the relative phase between the initial state amplitudes $a_{1}$ and $a_{3}$. This relative phase could be established by optical pumping into state $|1\rangle$ and using a Raman pulse to create the $|1\rangle$ $-|3\rangle$ state coherence. An equilibrium ensemble consists of molecules with various relative phases of populated states related to different vibrational modes. Following this statement we calculated coherences $\left|\rho_{12}\right|$ and $\left|\rho_{34}\right|$ as an average over relative phases between initially populated states $|1\rangle$ and $|3\rangle$. For $r=1,\left|\rho_{12}\right|$ and $\left|\rho_{34}\right|$ are shown in Fig. 3(a) (bold dashed and bold solid lines, respectively), as a function of the dimensionless intensity of the ultrafast laser pulse. Phase averaging is equivalent to calculating $\left|\rho_{34}\right|$ and $\left|\rho_{12}\right|$ as a sum of two contributions resulting from two initial population distributions: $\left(\rho_{11}=\frac{1}{2}, \rho_{33}=0\right)$ and $\left(\rho_{33}=\frac{1}{2}, \rho_{11}=0\right)$. This ap- 

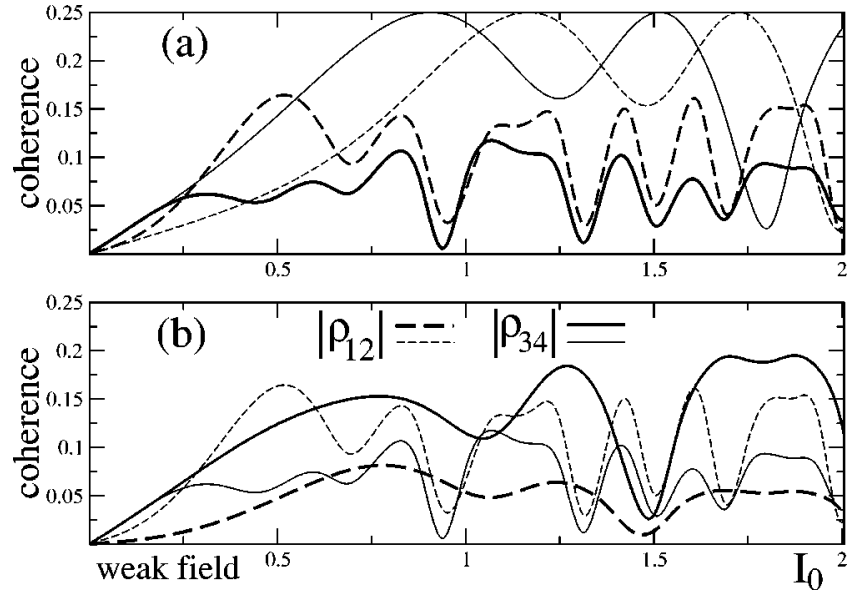

FIG. 3. Intensity dependence of the coherences of two, two-level systems; $\left|\rho_{12}\right|$ is shown by dashed lines and $\left|\rho_{34}\right|$ by solid lines. In (a) bold curves depict the case for $r=1$ corresponding to two coupled two-level systems with equal coupling constants; thin curves depict coherences for two independent two-level systems. The phase averaged solution gives much lower values of coherences than that for zero phase and coupling. In (b) bold curves show coherences for $r=1 / 2$, and thin curves for $r=1$. Weak coupling constants of the 1-2 system result in efficient population flow toward the 3-4 system, strongly coupled to the field.

proach eliminates the role of the phase between initially populated states. Coherences as a function of the intensity of the field calculated in such a way are identical to those in Fig. 3(a). Also shown in Fig. 3(a) are values of $\left|\rho_{34}\right|$ and $\left|\rho_{12}\right|$ (represented by thin solid and dashed lines, respectively), when there is no coupling between the two, two-level systems [obtained formally by setting $r^{2}=1, r=0$ in Eq. (1)]. From a comparison of the two sets of curves it is seen that phase averaged solution for two coupled two-level systems gives much lower values of $\left|\rho_{12}\right|$ and $\left|\rho_{34}\right|$ than that for uncoupled systems. In weak fields the coherence $\left|\rho_{34}\right|$ prevails over $\left|\rho_{12}\right|$, which is in agreement with Eq. (4). The coherence $\left|\rho_{12}\right|$ increases faster than that for the case of two uncoupled two-level systems. In strong fields $\left|\rho_{12}\right|$ and $\left|\rho_{34}\right|$ oscillate synchronously. The coherence $\left|\rho_{12}\right|$ almost always is larger than $\left|\rho_{34}\right|$ owing to its slightly smaller transition frequency. According to Fig. 3(a), in a molecule in weak fields a vibration at $\omega_{4}$ is excited stronger than that at $\omega_{2}$, regardless to the strength of coupling between two vibrational modes. Strong fields induce an efficient population transfer between all four levels in a molecule having nonzero mode coupling, causing excitation of both vibrations. Consequently, the amplitude of the induced electric dipole moment is modulated at both vibrational frequencies, giving rise to two Raman fields.

Numerical calculations show that $I(t)$ may result in significant differences between $\left|\rho_{12}\right|$ and $\left|\rho_{34}\right|$ in a system with different coupling constants $\mu_{i}$ which give different oscillator strength of the transitions. For $r=\frac{1}{2}$ the dependence of $\left|\rho_{34}\right|$ and $\left|\rho_{12}\right|$ on the intensity of the field is shown in Fig. 3(b) by bold solid and bold dashed lines, respectively. For such coupling constants, the probability of population transfer between two-level systems is equal to $1 / 2$, between states $|1\rangle$ and $|2\rangle$ it is equal to $1 / 4$, and between states $|3\rangle$ and $|4\rangle$ to
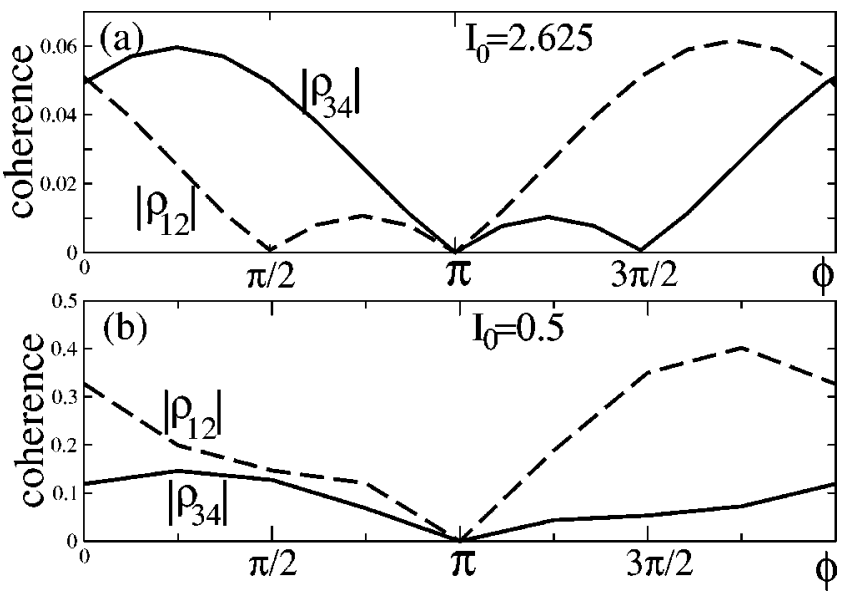

FIG. 4. Coherence of the 1-2 and 3-4 two-level systems as a function of initial relative phase between levels $|1\rangle$ and $|3\rangle$ for $r=1$ and equal initial populations of these levels. (a) $I_{0}=2.625 \pi$, (b) $I_{0}=0.5 \pi$.

1. As the result, population flows from the 1-2 to the 3-4 two-level system, maximizing coherence $\left|\rho_{34}\right|$.

For various values of intensity of the field $I_{0}$ the dependence of $\left|\rho_{12}\right|$ and $\left|\rho_{34}\right|$ on the relative phase between initially populated states $|1\rangle$ and $|3\rangle$ has been considered. In Fig. 4(a) the case for $I_{0}=2.625 \pi, r=1$ is represented (which gives almost equal values of $\left|\rho_{12}\right|$ and $\left|\rho_{34}\right|$ for the phase averaged solution). For phases from zero to $\pi,\left|\rho_{34}\right| /\left|\rho_{12}\right|$ $>1$, and a vibrational mode having frequency $\omega_{4}$ is excited stronger in a molecule than that having frequency $\omega_{2}$. For phases from $\pi$ to $2 \pi,\left|\rho_{34}\right| /\left|\rho_{12}\right|<1$ indicating the reverse picture of the strength of excitation of vibrational modes. Figure 4(a) shows that using phase control of the initially populated states allows one to enhance the coherence between desired vibrational levels in a molecule. The behavior of $\left|\rho_{34}\right| /\left|\rho_{12}\right|$ is sensitive to the intensity of field. For example, when $I_{0}=0.5 \pi$ [which gives a phase averaged solution $\left|\rho_{12}\right|=0.163$ and $\left|\rho_{34}\right|=0.059$ in Fig. 3(a)], the phase dependent calculation shows that $\left|\rho_{12}\right| /\left|\rho_{34}\right|>1$ for all phases except for $\phi=\pi$, see Fig. 4(b). The maximum value of coherence $\left|\rho_{12}^{\max }\right|=0.4$ at $\phi=7 \pi / 4$ is much higher than that for the phase averaged solution. This result demonstrates that in a molecular media with prepared relative phase between initially populated states an abnormally high polarization may be induced through the interaction with intense laser pulse. When the initial relative phase $\phi$ is equal to $\pi$, coherences $\left|\rho_{12}\right|$ and $\left|\rho_{34}\right|$ are equal to zero. This is the case for any external field. Populations of all states exhibit no time evolution. This result indicates an existence of a dark state, as can be deduced directly from the Hamiltonian in Eq. (1). For an arbitrary value $r$, the necessary conditions for a dark state are $n_{3} / n_{1}=r^{2}$ and $\phi=\pi$.

\section{SUMMARY}

We presented a semiclassical model of the coherent control of excitation of Raman transitions in two coupled twolevel systems using a broadband shaped laser pulse. We analyzed the effects caused by the coupling between four levels 
via a laser field. The coupling is shown to cause an efficientpopulation transfer between two-level systems. Effects caused by the relative phase between the initially populated states were analyzed. Depending on the intensity of the field, the initial phase may cause predominance of the coherence of one two-level system with respect to another. When the relative phase between initially populated levels $|1\rangle$ and $|3\rangle$ is equal to $\pi$, two, two-level systems encounter stationary conditions regardless of the external field strength, implying the existence of a molecular dark state. These results may be useful for investigation of decoherence processes caused by the driving field.

\section{ACKNOWLEDGMENTS}

The authors acknowledge financial support from the National Science Foundation (Grant No. PHY-9987916 and No. PHY-0244841) through the Center for Frontiers in Optical Coherent and Ultrafast Science (FOCUS) and the U. S. Office of Army Research under Grant No. DAAD19-00-10412. This work was partially supported by the National Science Foundation through a grant for the Institute for Theoretical Atomic, Molecular, and Optical Physics at Harvard University and Smithsonian Astrophysical Observatory.
${ }^{1}$ A. H. Zewail, J. Phys. Chem. A 104, 5660 (2000).

${ }^{2}$ V. Blanchet, M. Z. Zgierski, T. Seideman, and A. Stolow, Nature (London) 401, 52 (1999).

${ }^{3}$ E. Gershgoren, R. A. Bartels, J. T. Fourkas, R. Tobey, M. M. Murnane, and H. C. Kapteyn, Opt. Lett. 28, 361 (2003).

${ }^{4}$ J. L. Herek, W. Wohlleben, R. J. Cogdell, D. Zeidler, and M. Motzkus, Nature (London) 417, 533 (2002).

${ }^{5}$ T. C. Weinacht, R. Bartels, S. Backus, P. H. Backsbaum, B. Pearson, J. M. Geremia, H. Rabitz, H. C. Kapteyn, and M. M. Murnane, Chem. Phys. Lett. 344, 333 (2001).

${ }^{6}$ A. M. Weiner, D. E. Leaird, G. P. Wiederrecht, and K. A. Nelson, Science 247, 1317 (1990).

${ }^{7}$ N. Dudovich, D. Oron, and Y. Silberberg, Nature (London) 418, 512 (2002).

${ }^{8}$ S. H. Pullen, N. A. Anderson, L. A. Walker, and R. J. Sension, J. Chem. Phys. 108, 556 (1998)

${ }^{9}$ A. Hofmann and R. de Vivie-Riedle, J. Chem. Phys. 112, 5054 (2000).

${ }^{10}$ D. Oron, N. Dudovich, D. Yelin, and Y. Silberberg, Phys. Rev. Lett. 88, 063004 (2002).

${ }^{11}$ L. Dhar, J. A. Rogers, and K. A. Nelson, Chem. Rev. (Washington, D.C.) 94, 157 (1994).

${ }^{12}$ S. A. Rice and M. Zhao, Optical Control of Molecular Dynamics (Wiley, New York, 2000).

${ }^{13}$ P. W. Brumer and M. Shapiro, Principles of the Quantum Control of Molecular Processes (Wiley, New York, 2003).

${ }^{14}$ M. A. Nielsen and I. L. Chuang, Quantum Computation and Quantum Information (Cambridge University Press, Cambridge, 2001).

${ }^{15}$ A. Beige, D. Braun, B. Tregenna, and P. L. Knight, Phys. Rev. Lett. 85, $1762(2000)$

${ }^{16}$ S. A. Malinovskaya, P. H. Bucksbaum, and P. R. Berman, Phys. Rev. A 69, 013801 (2004). 